\title{
A family of quantization based piecewise linear filter networks
}

\section{Sørensen, John Aasted}

\section{Published in:}

I E E E International Conference on Acoustics, Speech and Signal Processing. Proceedings

Link to article, DOI:

10.1109/ICASSP.1992.226053

Publication date:

1992

Document Version

Publisher's PDF, also known as Version of record

Link back to DTU Orbit

Citation (APA):

Sørensen, J. A. (1992). A family of quantization based piecewise linear filter networks. I E E E International Conference on Acoustics, Speech and Signal Processing. Proceedings, 2, 329-332.

https://doi.org/10.1109/ICASSP.1992.226053

\section{General rights}

Copyright and moral rights for the publications made accessible in the public portal are retained by the authors and/or other copyright owners and it is a condition of accessing publications that users recognise and abide by the legal requirements associated with these rights.

- Users may download and print one copy of any publication from the public portal for the purpose of private study or research.

- You may not further distribute the material or use it for any profit-making activity or commercial gain

- You may freely distribute the URL identifying the publication in the public portal

If you believe that this document breaches copyright please contact us providing details, and we will remove access to the work immediately and investigate your claim. 


\title{
A FAMILY OF QUANTIZATION BASED PIECEWISE LINEAR FILTER NETWORKS
}

\author{
John Aasted Sørensen, Electronics Institute, Build 349, Technical University of Denmark, Lyngby
}

DK-2800 Denmark

\begin{abstract}
A family of quantization based piecewise linear filter networks is proposed. For stationary signals a filter network from this family is a generalization of the classical Wiener filter with an input signal and a desired response.

The construction of the filter network is based on the following elements:

Quantization of the input signal $x(n)$ into quantization classes. To each quantization class is associated a linear filter. The filtering at time $n$ is carried out by the filter belonging to the actual quantization class of $x(n)$ and the filters belonging to the neighbor quantization classes of $\mathbf{x}(n)$ (regularization).

This construction leads to a 3-layer filter network. The first layer consists of the quantization class filters for the input signal. The second layer carry out the regularization between neighbor quantization classes and the third layer constitutes a decision of quantization class from where the resulting output is obtained.
\end{abstract}

\section{INTRODUCTION}

In the following, a family of quantization based piecewise linear filter networks is proposed. For stationary signals a filter network from this family is a generalization of the classical Wiener filter [1] with an input signal $x(n)$ and desired response $d(n)$.

The object of the filter network is the approximation of a possible nonlinear function $G(n)$ between $x(n)$ and $d(n)$ as shown in Fig. 1.

The quantization based piecewise linear filter network shown in Fig. 2 is based on the assumption that the nonlinear relation $G(n)$ between $x(n)$ and $d(n)$ shown in Fig. 1, can be approximated by a set of linear filters, where each of these filters is constrained to a certain part of the input space of $x(n)$ defined by

$$
\mathbf{x}^{\prime}(n)=(x(n), x(n-1), \ldots, x(n-N+1)) .
$$

Here transposition of $x$ is denoted by $\mathbf{x}^{\prime}$.

Among others the quantization of the input signal $x(n)$ is characterized by the number of quantization classes obtained. If the number of classes is high (fine quantization), it is expected that the approximation of $G(n)$ will be accurate at the expense of a long adaptation time for the filters. However if the number of quantization classes is reduced (coarse quantization), the approximation error will increase and the adaptation time will be reduced because now there are fewer filters in the network.

The function of the filter network shown in Fig. 2 is as follows:

The input signal $x(n)$ is transferred to the quantizer

$$
j=\mathcal{Q}(\mathbf{x}(n), t h x)
$$

with threshold thx, which determines the current quantization class $\mathbf{j}$, where $1 \leq j \leq|\mathcal{Q}(n)|$. The number of quantization classes $|\mathcal{Q}(n)|$ vary with time and the quantization class filters constitutes the first layer in the filter network.

Then the set of neighbor quantization classes

$$
\mathcal{N}(j, t h n)
$$

of the current quantization class $\mathrm{j}$ is determined, using the threshold thn. Here $|\mathcal{N}(j)|$ denotes the number of neighbor classes of quantization class $j$. This defines the second layer of linear filters used for regularization. Finally the third layer constitutes a decision among the possible quantization classes.

The following two different filtering functions are defined:

$$
\hat{d}_{1}(n)=\frac{1}{|\mathcal{N}(j)|+1}\left\{\mathbf{w}_{j}^{\prime}+o n e_{j}^{\prime} \mathbf{v}_{j}^{\prime}(n)\right\} \mathbf{x}(n)
$$

or

$$
\hat{d}_{2}(n)=\frac{1}{|\mathcal{N}(j)|+1} \mathbf{w}_{j}^{\prime}\left\{\mathbf{I}+\mathbf{V}_{j}(n) \mathbf{V}_{j}^{\prime}(n)\right\} \mathbf{x}(n)
$$

The rows of the neighbor filter matrix $\mathbf{V}_{j}^{\prime}$ are filtervectors of the neighbor classes of quantization class $j$. one $_{j}^{\prime}$ is a row vector with a number of ones equal to the number of neighbor classes of $j$ and $I$ is the identity matrix. 
Thus $\hat{d}_{1}(n)$ is the mean value of the filtering results of $w_{j}(n)$ and the neighbor filters of the quantization class $j$.

In $\hat{d}_{2}(n)$ the neighbor filter results $\mathbf{w}_{k}^{\prime} \times(n)$ are weighted with $w_{j}^{\prime} w_{k}$. This weighting emphasize neighbor filters with approximately the same direction as the quantization class filter and reduce the response from filters approx. orthogonal to the quantization class filter. Several functional forms, beyond the above two, of the neighbor filter responses can be defined.

\section{PIECEWISE LINEAR FILTERING ALGORITHM}

In the following are given the main elements of the piecewise linear filtering algorithm:

Input signals

$x(n)$ : Input signal.

$N$ : Dimension of input signal space.

$\mathbf{x}^{\prime}(n)=(x(n), x(n-1), \ldots, x(n-N+1))$.

$d(n)$ : Desired response.

Quantization classes

$j=Q(\mathbf{x}(n), \operatorname{th} x):$ Quantization class no. $j$

$t h x$ : Quantization threshold.

$|\mathcal{Q}(n)|:$ The number of quantization classes.

$\mathcal{Q}=\left\{\mathbf{q}_{1}, \ldots, \mathbf{q}_{|\mathcal{Q}(n)|}\right\} ;$ Set of quantization classes of $\mathbf{x}(n)$.

$\operatorname{dist}\left(\mathbf{x}(n), \mathbf{q}_{k}\right)$ : Distance between $\mathbf{x}(n)$ and $\mathbf{q}_{k}$.

\section{Neighbor classes}

$\mathcal{N}(j, t h n):$ Set of neighbor classes of quantization class no. $j$.

thn: Neighbor class threshold.

$|\mathcal{N}(j)|$ : The number of neighbor classes of quantization class no. $j$.

$\mathcal{N}=\{1,2, \ldots,|\mathcal{Q}(n)|\}:$ Set of quantization class numbers.

$\mathcal{N}(j, t h n) \subseteq \mathcal{N}$.

Filtering

$\mathbf{w}_{j}^{\prime}$ : Filter of quantization class no. j. $1 \leq j \leq|\mathcal{Q}(n)|$. $\mathbf{w}_{j}^{\prime}(n)=\left(w_{1, j}(n), w_{2, j}(n), \ldots, w_{N, j}(n)\right)$

$\mathbf{v}_{j}^{\prime}(n)$ : Filtermatrix of neighbor classes of quantization class no. $j$.

$\mathrm{V}_{j}^{\prime}(n) \in \mathcal{R}^{|\mathcal{N}(j)| \times \mathcal{N}}$
$\mathbf{V}_{j}^{\prime}(n)=\left(\begin{array}{c}\vdots \\ w_{r}^{\prime}(n) \\ \vdots\end{array}\right)$ where $r \in \mathcal{N}(j, t h n)$

one $_{j}^{\prime}=(\underbrace{1,1, \ldots, 1}_{|\mathcal{N}(j)|})$.

I: $N \times N$ identity matrix.

Quantization class algorithm

Result: $j=\mathcal{Q}(\mathbf{x}(n), t h x)$.

if $n=1$

then $\mathbf{q}_{1}=\mathbf{x}(1)$

$|\mathcal{Q}(1)|=1$

else $j=0$

for $i=1,2, \ldots,|\mathcal{Q}(n)|$

if $\operatorname{dist}\left(\mathbf{x}(n), \mathbf{q}_{i}\right) \leq t h x$ then $j=i$

endfor

if $j=0$

then $|\mathcal{Q}(n)|=|\mathcal{Q}(n)|+1$

$$
j=|\mathcal{Q}(n)|
$$

endif

$$
\mathbf{q}_{j}=\mathbf{x}(n)
$$

endif

\section{Neighbor class algorithm}

Result: $\mathcal{N}(j, t h n)=$ Neighbor classes of $j$.

Start searching for neighbor classes of quantization class $j$ :

$\mathcal{N}(j, t h n)=$ empty set

for $i=1, \ldots,|\mathcal{Q}(n)|$

if $\operatorname{dist}\left(\mathbf{q}_{i}, \mathbf{q}_{j}\right) \leq t h n$ endif

$$
\text { then } \mathcal{N}(j, \overline{t h} n)=\mathcal{N}(j, t h n) \cup\{i\}
$$

endfor

Piecevise Linear Filtering Algorithm

Result: $\hat{d}(n), \operatorname{err}(n)$

for $n=1,2, \ldots$

quantization class $j=\mathcal{Q}(\mathbf{x}(n), t h x)$

neighbor classes of $j: \mathcal{N}(j, t h n)$

quantization class filter $\mathbf{w}_{j}(n)$

neighbor class filters $\mathbf{v}_{j}^{\prime}(\boldsymbol{n})$

$\hat{d}(n)$ (either $\hat{d}_{1}(n)$ or $\hat{d}_{2}(n)$ )

$\operatorname{err}(n)=d(n)-\hat{d}(n)$

estimation of $w_{j}(n+1)$

endfor 


\section{INITIAL INVESTIGATION}

The proposed quantization based piecewise linear filter network is being investigated by analysis and simulation. The main object of the analysis is to compare the filter network properties with the classical adaptive filters [1], the filters from [2] and [3] and the well known 3-layer feed-forward neural networks [4]. The filter network has a structural relationship to a 3-layer feed-forward neural network, where the filter network parameters: $N, t h x$ and $t h n$, are related to the feedforward neural network parameters: The number of input nodes, the number of nodes in the hidden layer and the connectivity between the hidden layer and the output layer.

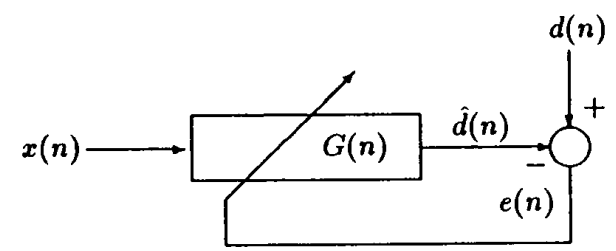

\section{REFERENCES}

1. S. Haykin, "Adaptive Filter Theory", 2. Ed Prentice-Hall, 1991.

2. Ji-Nan Lin, Rolf Unbehauen, "Adaptive Nonlinear Digital Filter with Canonical Piecewise-Linear Structure".

IEEE Trans. CAS-37, March 1990.

3. Saul B. Gelfand, C.S. Ravishankar, Edward J. Delp "A Tree-Structured Piecewise Linear Adaptive Filter".

IEEE ICASSP-91 pp.2141-2144.

4. J. Hertz, A. Krogh, R.G. Palmer "Introduction to the Theory of Neural Computation"

Addison-Wesley Publishing Company, 1991.

Figure 1: Adaptive filter modelling a nonlinear function $G(n)$

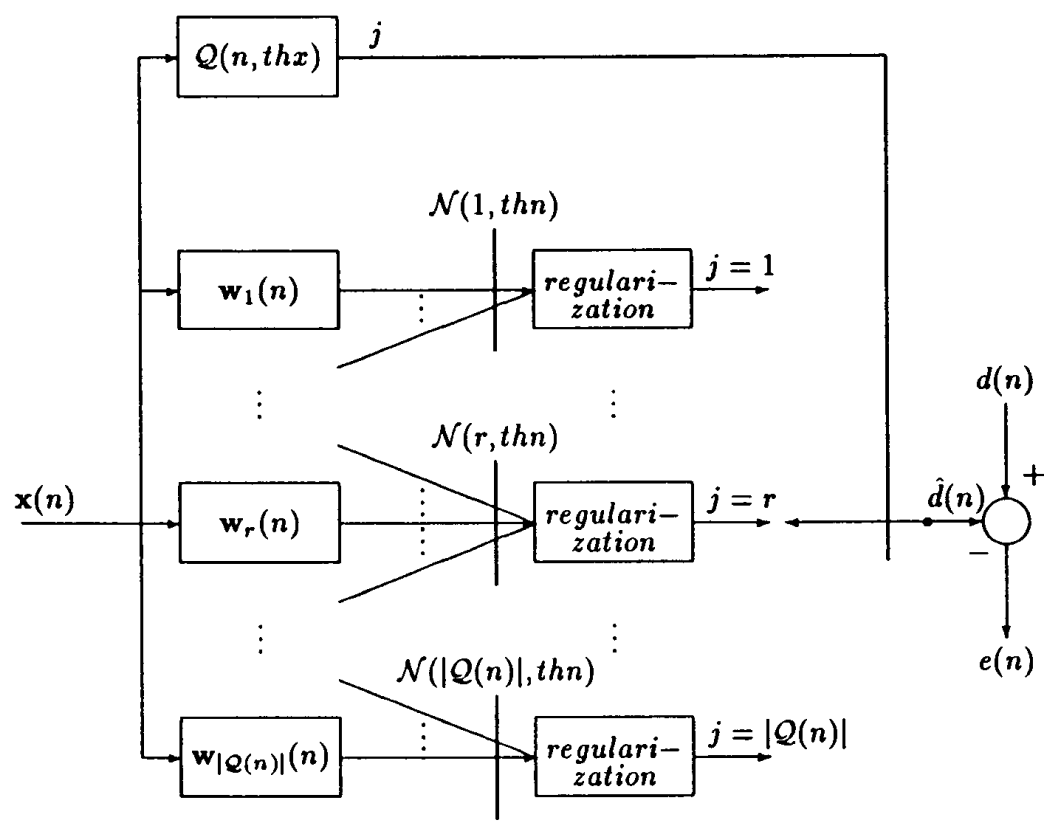

Figure 2: Quantization based piecewise linear filter network

\section{II-331}

